\title{
Identifying patients at risk of deterioration in the Joint Emergency Department
}

\author{
Thomas Schmidt • Uffe Kock Wiil
}

Received: 17 September 2014 / Accepted: 26 January 2015/Published online: 22 February 2015

(C) The Author(s) 2015. This article is published with open access at Springerlink.com

\begin{abstract}
In recent years, Danish hospitals have merged their emergency facilities into Joint Emergency Departments. This poses new collaborative challenges across traditionally separated specialized departments, which now have to collaborate in a shared environment. Despite established protocols and patient monitoring regimes, as many as $31 \%$ of non-critical patients deteriorate with potentially severe consequences. To understand why this may be, we conduct a field study with the aim of investigating what factors external to patients relate to the clinicians' ability to notice adverse events. We look at the case through the lenses of common information spaces. In particular, we apply Bossen's seven-parameter framework to discover new dimensions of how Emergency Departments and individual clinicians identify and respond to unforeseen events, and how they handle the associated cognitive challenges. We complement these findings with a review of a novel taxonomy for patient monitoring. Our contribution is the identification of four improvement areas for patient monitoring platforms in terms of support for the identification of patient deterioration.
\end{abstract}

Keywords Health informatics - CSCW - Awareness · Shared cognition - Common information spaces . Patient deterioration · Joint Emergency Departments

T. Schmidt (两) · U. K. Wiil

The Maersk Mc-Kinney Moller Institute, University of Southern Denmark, Campusvej 55, 5230 Odense M, Denmark

e-mail: schmidt@mmmi.sdu.dk

U. K. Wiil

e-mail: ukwiil@mmmi.sdu.dk

\section{Introduction}

The treatment of emergency patients in Denmark has recently been restructured by merging multiple specialties into a single point of entry at the hospital, known as Joint Emergency Departments (JEDs). As a consequence of this shift, members of the various specialties at the hospitals have been required to collaborate in new ways as a range of departments now contribute in providing specialty resources to the JEDs. In this setting, $31 \%$ of admitted patients with non-critical vital values experience deterioration within the first $24 \mathrm{~h}$ of hospitalization (Henriksen et al. 2014). These patients were found to have an increase in 30-day mortality by a factor of four. In fact, any increase in clinically relevant parameters is associated with a worse survival prognosis (Kellett et al. 2011). Yet, it is difficult to spot patients at risk of deterioration without the aid of formalized monitoring programs. A lot of efforts have been put into trying to evaluate and improve the Early Warning Scoring (EWS) systems implemented in hospital practices (Gao et al. 2007). However, there appear to be generic challenges present in all systems as each system provides some improvement, but generally fails to adequately solve the situation at all necessary levels (Brabrand et al. 2010). While it is evident that the accuracy of EWS systems plays a huge role in identifying adverse events in due time, and that the discriminative power of these systems can be improved, several findings in the literature indicate that human and organizational factors are equally important. For instance, partial and inconsistent adherence to established vital signs registration protocols have been observed during handovers (Hands et al. 2013). Likewise clinical experience has been shown to hugely influence the ability to predict mortality (Brabrand et al. 2014). We thus assume that at least part of the cause for not identifying deterioration in due time also lies within the 
Emergency Department's (ED's) organization of collaborative work itself. This motivates seeking an answer to the overall research question of 'what organizational and technical factors may hinder the identification of patients at risk of deterioration in the JED?' To answer this question, we investigate the interplay between patient treatment and knowledge work in a Danish JED.

In EDs, clinicians are forced to make critical decisions in very short time frames with large degrees of uncertainty, and thus, the accumulation and transfer of knowledge are fundamental when dealing with the issue of identifying adverse events. In the borderland between people, their technology, and the surrounding organization, clinicians face a sizeable cognitive challenge in dealing with a high diversity in illnesses and patients. Healthcare services are, by the nature of their complexity, necessarily cooperative. Thus, technology and clinical work have become inseparable entities and have as such been a favored field for Computer-Supported Cooperative Work (CSCW) researchers, seeking to identify efficiency improvements (Fitzpatrick and Ellingsen 2013). In this article, we draw upon the existing body of work from CSCW, and in particular the concept of common information spaces (CISs; Bannon and Bødker 1997), and elaborated upon with Bossen's seven-parameter framework to assess the interplay between technology and work (Bossen 2002). Through this approach, we uncover the collaborative characteristics of the department via a 5-month field study and highlight the vitally important pathways of sharing knowledge and information.

Today, there is now an increasing demand to optimize the interplay between healthcare organizations and their health information technologies as more medical procedures are automated and information sharing is becoming paperless. This forces medical device manufacturers to focus on means of integration into health informatics technology infrastructure and the surrounding organization. This shift is in line with the priorities of this paper, as we wish to investigate the relationship between clinicians and their use of technology in identifying deteriorating patients. To support our findings, we propose a simple taxonomy for evaluation and classification of patient monitoring platforms.

The contribution of this paper is the identification of a number of implications for work and cooperative practices, which we identify as being needed to improve the identification of patients at risk of deterioration. The paper starts by describing the background for our theoretical framework (Sect. 2). This is followed by a review of related work in the context of EDs and EWSs in order to situate our work in relation to existing research (Sect. 3). We proceed to describe the settings of the JED (Sect. 4) and our methodology with a focus on the design of the field work (Sect. 5). With the triad of background information, related work, and methodology, we proceed to identify the findings (Sect. 6), from which we provide a discussion (Sect. 7). Limitations of the presented work are described in Sect. 8. Finally, Sect. 9 provides our conclusions and a presentation of future work opportunities.

\section{Background}

Computer-Supported Cooperative Work's concern with the interplay between people in organizations and information technology in a collaborative work environment, involve important entities such as people, artifacts, and settings, and how these work together (Schmidt and Simone 1996). Schmidt and Simone proposed a distinction between cooperative work and articulation work. Cooperative work is the settings, in which distributed actors collaborate through individual influence of the shared environment. This distribution instills a complexity due to the necessary alignment of activities. Articulation work is the effort required to bring this distributed work into line. Articulation work is essentially added work, and thus something which is sought reduced. Schmidt and Simone (1996) studied how artifacts could be used to shrink the need for articulation work, if the artifacts were dealt with a set of procedures and conventions. They coined this approach as coordinating mechanisms. These mechanisms became the starting point for Bannon and Bødker (1997), who felt that CSCW lacked a key concept with regard to the effort required to transform information into common knowledge in a shared space, and how this effort is coupled to the parties' mutual understanding of each other and the context (Bannon and Bødker 1997).

\subsection{Common information spaces}

Accordingly, Bannon and Bødker introduced the notion of a CIS as a construct for the way, in which people, artifacts, and settings are brought together to accomplish a given piece of work (Bannon and Bødker 1997). In this seminal paper, Bannon and $\mathrm{B} \varnothing \mathrm{dker}$ focused on the dialectical nature of CIS in the sense that the information space typically has both an open-ended perspective from where its participants share, change, and interpret the information from a backstage'/non-public environment, and, on the other side, a closed front-stage nature where information is represented as boundary objects with traits of immutability. Mapping this two-sided nature to the context of health care in hospitals, we find clinicians working under a set of requirements to adhere to the closed nature of the CIS they are operating in, but exert the majority of their coordinative work efforts through the open-ended aspects of the CIS. Bossen (2002) identified CIS as a valuable concept, but one 
that is illusive and difficult to apply consistently to research work. Thus, Bossen proposed seven parameters to provide a more concrete framework for understanding the characterization of a specific CIS:

1) The degree of distribution: How are persons and systems affected by their physical distribution across the workspace?

2) The multiplicity of webs of significance: How do different backgrounds, professions, and job descriptions affect the CIS? Heterogonous webs of significance require a higher degree of interpretation of communication to avoid misunderstandings.

3) The level of required articulation work: To what extent is continuous articulated coordination among the actors required to execute the tasks at hand?

4) The multiplicity and intensity of means of communication: What communication channels are used to support mutual understandings and interpretation of information? If there is a need for multiple channels, this is often a sign that continuous calibration of meaning is needed.

5) The web of artifacts: What artifacts are utilized in the CIS, and what is the nature of these artifacts? For example, open-ended artifacts such as free-text forms impose less standardization than check lists.

6) Immaterial mechanisms of interaction: What habits, divisions of labor, and other immaterial structures reduce the need for coordination?

7) The need for precision and promptness of interpretation: What is the need for informational precision and promptness of response to new information in our context?

\subsection{Alternative frameworks}

Common information spaces are not the only available framework for understanding the challenges of collaboration in complex environments. We find multiple similarities between Joint Cognitive Systems (JCSs; Johansson and Hollnagel 2006) and CIS. Both constructs seek to describe the challenges of information shaping and sharing across multiple actors. CIS emphasizes the importance of information richness and the interplay between different types of work, artifacts, and shared knowledge. In comparison, JCS focuses more on temporal aspects and the simultaneously enabling and limiting aspects of interacting systems in the context.

Although a synthesis of frameworks would be interesting, we focus on CIS and utilize Bossen's seven parameters of the CIS to explore our research question, as CIS positions itself closely with the characteristics of the JED's environment. To achieve this, we investigate the traits and effects of interplay between individual actors, time, and the environment. As the CIS is a product of its surrounding environment, understanding the context must be a first necessary step in trying to explain the CIS.

\section{Related work}

Although the literature on collaborative work in health care is quite comprehensive (Fitzpatrick and Ellingsen 2013), there is only a handful of CSCW papers that focus on the particular dynamics of an ED. While several papers in other research fields have focused on the predictive capabilities of patient assessment models, few have dealt with the issue of patient deterioration from the perspective of our research question.

Clinical intuition plays an important role in identifying patients at risk of deterioration (Odell et al. 2009). The ability of clinicians to predict patients at risk of dying during admission increased with experience, and their prediction had very good discriminative power (Brabrand et al. 2014). This improved even further if combined with the prediction from other groups and classification systems. This highlights Bossen's points that the clear distinction between groups of clinicians in terms of labor division between assistants, nurses, and physicians could be blurred by the differences in experience between clinicians (Bossen 2002). Evidently, the necessity of acquiring more insight into the competencies of colleagues than simply their profession is apparent; also, this insight can be utilized to increase the odds of identifying adverse events.

A workplace study of triage work practices found; firstly, that the key practice of prioritization in an ED is really a collaborative effort although the task is usually formally assigned to a single clinician; secondly, that triage work does not follow a standardized script (Bjørn and Rødje 2008). This can be seen as a disruption in the attempt to minimize articulation work by standardizing the operational procedures of the department. Finally, the nature of triage work is so such complex that it cannot be considered as a single event in the processing of patients.

EWS systems are often modified to specific clinical settings or local health policies. Three of the major systems in use today are the Modified Early Warning Score (MEWS; Subbe et al. 2001). The VitalPac EWS (ViEWS; Prytherch et al. 2010) and the derived National Early Warning Score (NEWS; Royal College of Physicians 2012). The implementation and evaluation of EWS systems ranges from paper-based tracking systems (Moon et al. 2011) to assisted calculations based on either automatic or manual registrations such as VitalPac, to fully automated systems (Dawes et al. 2014; Tarassenko et al. 2006). EWS systems are also known as 'track and trigger' (TT) systems, 
which describes the necessity of registering patient observations in regular intervals. TT systems can be grouped into two types: single-parameter systems, which trigger alarms on deviation from normality (Smith et al. 2008), and multi-parameter TT systems, which calculate a combined severity score (Gao et al. 2007). The main purposes of all these systems are essentially to support detection of patient deterioration. A shared trait is the matter that all systems depend on data collection and clinical interpretation. This interpretation is often explored in terms of situation awareness, which embodies the ability to maintain a representation of the surrounding environment (Schulz et al. 2013). Situation awareness is subdivided into three levels: perception, comprehension, and projection. Providing clinicians with a formalized system built on results from situation awareness studies in a pediatric hospital center, showed improvements in the identification of unrecognized clinical deterioration (Brady et al. 2013). A review of the properties of collaborative work in a trauma unit (Xiao et al. 2002) found that the degree of freedom to redefine interfaces should be present in collaborative coordination devices as unforeseen events often are best handled by improvisation. Similar research was conducted in the setting of an ED (Bjørn and Hertzum 2010), who introduced the concept of artifactual multiplicity to describe the way in which artifacts often serve multiple practices while playing an important role in maintaining relations between seemingly unrelated tasks.

While this mutual understanding of competencies and shared artifacts may further reduce the need for articulation work, Bardram and Bossen complemented the aspect of this concept, by introducing mobility work (Bardram and Bossen 2005a). As articulation work describes the effort needed to coordinate cooperative work, Bardram and Bossen argue that this notion mainly places emphasis on the temporal aspects of work, and thus neglects the influence of spatiality in a work environment. Bardram and Bossen describe mobility work as "the work needed to achieve the right configuration of people, resources, knowledge, and place in order to carry out tasks" (Bardram and Bossen 2005a). Strauss defined standard operational procedures (SOPs) as a mean to reduce articulation work by formalizing scheduling, task allocation, and flow of resources such as skills, time, energy, supplies, and equipment (Strauss 1985). Bardram and Bossen (2005a) provide a similar construct in terms of their standard operating configuration (SOC), which seeks to facilitate spatially distributed cooperative work with the intent of minimizing the mobility work effort. Yet, even though established protocols are in place, clinicians are still prone to adhering to them in suboptimal ways (Hands et al. 2013).

In summary, most deterioration studies focus on Intensive Care Units (ICUs) as these are already settings with the highest degree of supervision and patient monitoring. In ED settings, we have found several applications of CSCW research, but few focus on how to bring together human cognitive work and technological systems to deal with the specific challenges of patient deterioration.

\section{The settings}

In this section, we provide a description of the JED, and in line with (Weingart and Wyer 2006), which portrays the ED as a cognitive battlefield, we find a very complex and dynamic environment where a frictionless interplay between clinicians and information technology can make the difference between life and death. The settings are presented based on the available online descriptions of the JED, and our own observations and review of available IT systems and artifacts. This data triangulation approach is described more thoroughly in Sect. 5.

\subsection{The department}

The environment of our research is a newly established JED at Odense University Hospital, a large Danish university hospital with an uptake population of 288,000 citizens. JEDs are a new construct for hospitals in Denmark, but share many similarities with how EDs are structured in Englishspeaking countries. The JED consists of two floors: a ground floor with an Emergency Treatment Center (ETC), which handles the initial assessment and treatment of patients, and a second floor with a Center for Accelerated Patient flows (CAP). The ETC has two entries: a reception for patients who arrive by their own means and an ambulance entry for patients who require assistance, either because of their mobility state, or severity of arrival cause.

On the ground floor, we find receiving areas with triage and trauma rooms (area \#1 in Fig. 1), a waiting area next to the reception (area \#2), a treatment area with injury rooms where specialized nurses handle minor injuries (area \#3), an open observation area for both bedbound and sitting patients, and treatment rooms for patients who require more privacy and medical consideration (both included in area \#4). The ETC has an open office area for physicians (area \#5) and radiology facilities (area \#6).

The CAP on the second floor has capacity for 50 beds for shorter admission periods of up to $48 \mathrm{~h}$. The floor is organized into two medical (areas \#7 and \#8) and one surgical ward (area \#9). The center of each ward is an office space which is occupied mainly by nurses.

Patient monitors are available in the treatment rooms in the ETC and at each bed at the CAP. 


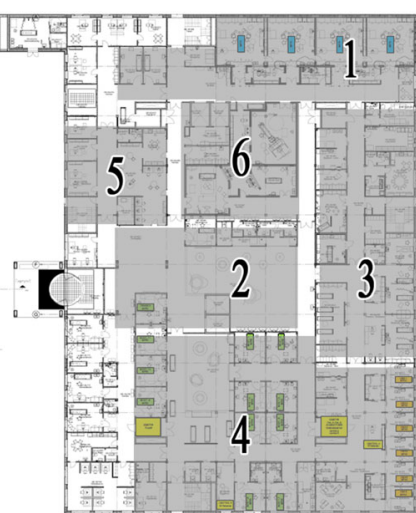

Emergency Treatment Center

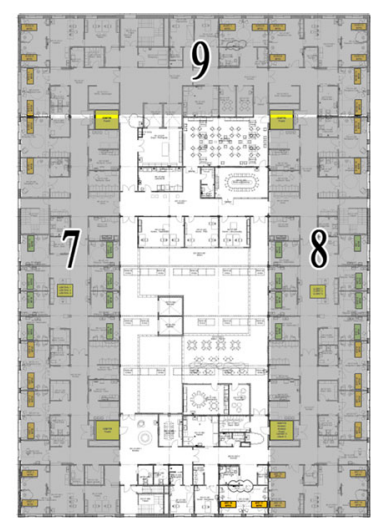

Center for Accellerated Patient flows
Fig. 1 JED overview

\subsection{Information technology in use}

At the department, we find several software systems used for both distinct and overlapping purposes, and of these, we focus on four systems which greatly impact the daily clinical workflows at the department.

Firstly, the entire hospital relies on a Patient Administration System (PAS) for transfers and financial registration. Secondly, the Cosmic ${ }^{1}$ Electronic Health Record (EHR) system, which is used throughout the entire region, thirdly the JED utilizes the Cetrea ${ }^{2}$ logistics system for tracking the flow and state of patients in the department, and lastly the vital signs of all patients admitted to beds can be monitored via a Philips IntelliVue system ${ }^{3}$ using mainly MP30 and MP50 monitors.

While the PAS system is mainly used for administrative purposes, the three other systems are shared among all clinical work groups in the department. The Cetrea logistics system offers a glance-able overview of the state of all patients in the system at the department, with differentiated views for the ground floor physician coordination room and for the five other ward offices and thus acts as the departmental whiteboards. The Cetrea logistics system greatly aid internal communication and coordination as the system builds a web of coordinative artifacts as described by Bardram and Bossen (2005b). Physicians, nurses, and assistants all rely on the EHR system for registering clinical observations, and each clinical group accesses specific registration forms and data structures in the system according to their responsibility domain. The EHR system also integrates with multiple laboratory systems.

\footnotetext{
${ }^{1}$ http://www.cambio.se/.

2 http://www.cetrea.com/.

${ }^{3}$ http://www.healthcare.philips.com/.
}

\subsection{The staff and organization of work}

Due to the scope of our research, the field study mainly focused on staff directly involved in the treatment of patients. The clinical side of the Danish healthcare sector is comprised of three major groups of workers: assistants, nurses, and physicians.

The JED is manned by junior and senior physicians. Broadly speaking, physicians are in charge of building a diagnosis based on the presented symptoms, prescribing treatments, and conducting patient consultations. Nurses provide first hand patient care and comfort, including administration of medications, patient hygiene, and facilitating the overall recovery of the patient. CAP also employs a number of assistants; their role is essentially similar to that of the nurses, except that an assistant is not authorized to handle administration of medications to patients.

The planning and organization of work is achieved in ways similar to most other hospital departments in terms of teams and fixed shifts. All clinicians are organized into teams, primarily to ensure staff accountability and empowerment. Nurses on the CAP are organized into ward-specific teams. The ETC has two teams, and the CAP is run by three teams. A work day is divided into three nurse shifts: a day shift from 7 a.m. to 3 p.m., an evening shift from 3 p.m. to 11 p.m., and a night shift from 11 p.m. to 7 a.m. In a similar manner, the medical physicians are organized into two teams with distinct responsibilities. Each team covers a particular part of the CAP, sees to certain groups of patients in the ETC, and responds to certain event types such as trauma calls or cardiac arrests.

\subsection{Roles at work}

Besides engaging multiple professions, the organizational structure of the JED also defines a set of roles which formalize internal coordination and work scope.

The overall responsibility for the patient flow and its optimization is handled by the flow master role which is assigned to a JED senior physician who masters both the clinical and organizational challenges. The flow master ensures that the patient is registered in the logistics system and that all necessary information has been gathered.

The flow master has the final say in disagreements regarding the triage level of a patient and is thus in control of the handling of ETC patients. Nursing care is managed by a coordinating nurse on each floor. Their responsibility is to ensure that the patients receive both competent and qualified care. Consequently, the coordinating nurse simultaneously acts as a supervisor for other nurses and keeps track of the patient-related logistics. The coordinating nurse is in charge of synchronizing patient transfers with all collaborating departments, and internally in the JED department between the ETC and the CAP. 
Fig. 2 ADAPT triage model

\begin{tabular}{|c|c|c|c|c|c|}
\hline & $\begin{array}{c}\text { Triage-1 } \\
\text { Time: } 0 \text { min } \\
\text { Resuscitation } \\
\text { Continous } \\
\text { observation }\end{array}$ & $\begin{array}{c}\text { Triage-2 } \\
\text { Time: } 15 \text { min } \\
\text { Stabilization } \\
\text { Monitoring }\end{array}$ & $\begin{array}{l}\text { Triage-3 } \\
\text { Time: } 60 \text { min } \\
\text { Less urgent } \\
\text { Supervision }\end{array}$ & $\begin{array}{l}\text { Triage- } 4 \\
\text { Time: } 180 \mathrm{~min} \\
\text { Not urgent } \\
\text { Reassess }\end{array}$ & $\begin{array}{l}\text { Triage-5 } \\
\text { Time: } 240 \text { min } \\
\text { Fast-Track } \\
\text { Reassess }\end{array}$ \\
\hline Airways & $\begin{array}{l}\text { No free airway } \\
\text { inspiratory stridor }\end{array}$ & $\begin{array}{l}\text { Airways threatned } \\
\text { Changed voice }\end{array}$ & $\begin{array}{l}\text { Irritated throat } \\
\text { Normal voice }\end{array}$ & Free airways & \begin{tabular}{|l|} 
Objectively \\
unaffected ano \\
no vital values \\
\end{tabular} \\
\hline Breathing & $\begin{array}{l}\text { Heavy respiratory } \\
\text { insufficiency } \\
\mathrm{SpO}_{2}<80 \% \\
8>\mathrm{RF}>35 \\
\end{array}$ & $\begin{array}{l}\text { Resting Dyspnea } \\
\mathrm{SpO}_{2}: 80-89 \% \\
\mathrm{RF}: 30-35\end{array}$ & $\begin{array}{l}\text { Functional } \\
\text { Dyspnea } \\
\mathrm{SpO}_{2}: 90-94 \% \\
\text { RF: } 25-29 \\
\end{array}$ & $\begin{array}{l}\text { Normal } \\
\mathrm{SpO}_{2} \geq 95 \% \\
\text { RF: } 8-24\end{array}$ & \begin{tabular}{|l|} 
Objectively \\
unaffected and \\
no vital values
\end{tabular} \\
\hline Circulation & $\begin{array}{l}\text { Pulse (reg) }>130 \\
\text { Pulse (irreg) }>180 \\
\mathrm{BT}_{\text {sys }}<80\end{array}$ & $\begin{array}{l}40>\text { Pulse }>120 \\
\mathrm{BT}_{5 y s} \leq 90\end{array}$ & \begin{tabular}{|l|} 
Pulse: $111-120$ \\
or \\
Pulse: $40-49$ \\
\end{tabular} & Pulse: $50-110$ & \begin{tabular}{|l|} 
Objectively \\
unaffected ano \\
no vital values \\
\end{tabular} \\
\hline Disability & $\begin{array}{l}\mathrm{GCS}^{1} \leq 8 \\
\text { OBS pupil } \\
\text { dialation } \\
\text { Cramps / Status } \\
\text { EP }\end{array}$ & $\begin{array}{l}\text { GCS: } 9-13 \\
\text { Very agitated } \\
\text { Acute unaware }\end{array}$ & \begin{tabular}{|l|} 
GCS: 14 \\
Moderately \\
agitated
\end{tabular} & $\begin{array}{l}\text { CGS: } 15 \\
+ \text { normal pupils }\end{array}$ & \begin{tabular}{|l|} 
Objectively \\
unaffected ano \\
no vital values
\end{tabular} \\
\hline Exposure & $\begin{array}{l}\text { OBS Chemical } \\
\text { contamination? }\end{array}$ & $\begin{array}{l}\mathrm{T}_{\mathrm{p}}>40^{\circ} \mathrm{C} \\
\text { Hypotherm }<32^{\circ} \mathrm{C}\end{array}$ & $\begin{array}{l}T_{p}: 38,1-40^{\circ} \mathrm{C} \\
\text { or }<36^{\circ} \mathrm{C} \\
\text { (sepsis) }\end{array}$ & $T_{D}: 36-38^{\circ} \mathrm{C}$ & $\begin{array}{l}\text { Objectively } \\
\text { unaffected ano } \\
\text { no vital values } \\
\end{array}$ \\
\hline
\end{tabular}

\subsection{Work practices during patient admission}

Upon arrival at the JED, all patients are assessed and classified according to the five-level ADAPT triage model depicted in Fig. 2 (Lauritzen et al. 2009). The patient is scored on the ABCDE (Airways, Breathing, Circulation, Disability, Exposure) principles. The most severe of any score determines the triage category.

From 7 a.m. to 11 p.m., both front door entries are manned by a triage nurse. During the night shifts, triage is handled by the triage nurse in the reception area or delegated to the ETC coordinating nurse.

The department has defined a number of Care Continuity Plans (CCPs) which formalize the processing of patients at the JED. These plans are a way of categorizing patients in ranges from minor injuries not requiring hospitalization, up to severe multi-comorbid patients requiring immediate admission to the hospital. In addition to the CCPs, the department is in a process of formalizing Emergency Care Packages (ECPs) which intends to optimize the processing of a patient during the first $4 \mathrm{~h}$ of arrival. Thus, the ECPs simultaneously define a time schedule, a set of required investigations, and best practice quality assurance.

In the last phases of the initial triage, the triage nurse assigns an ECP to the patient; this information is registered in the Cetrea system by the nurse. If the nurse is in doubt about which package to assign, the flow master is consulted.

Following the triage, the patient is taken to the treatment area where the patient will be assigned either a chair or a bed by the team of treatment nurses. This placement is handled by the ETC coordinating nurse, who ensures that the bed capacity is best utilized and that the department has ample capacity to receive new patients.

During the first $4-8 \mathrm{~h}$ of admission, a patient will typically be relocated a couple of times due to examinations, reallocation of beds, and staff. If the senior physician assesses that the patient needs prolonged treatment, the flow master will admit the patient to the CAP. The relocation takes place when there is sufficient receiving capacity at CAP.

Throughout the admission period, the triage level of a patient determines the level of clinical observation required to ensure that the patient receives attention matching their situation. All patients have their vital values registered manually by a nurse in regular intervals dictated by triage level. Only critical patients are continuously attached to a monitor, but data are still manually registered in the EHR system.

\section{Methodology}

We designed our study as a qualitative single-site case description with the intent of seeking generalizability through transferability of our findings for other researchers. This approach is described as case-to-case transfer by Firestone (1993).

The findings and results in this article are based on an ongoing collaboration with the clinicians at the JED, and a field study spanning 5 months, during which the main author followed clinicians from all three staff groups: service assistants, nurses, and physicians during their work day. The field study consisted of 11 sessions of $8 \mathrm{~h}$ each. Our stopping criteria were set as a minimum coverage of all 
work groups (assistants, nurses, and physicians), roles (assistant, ward nurse, triage nurse, coordinating nurse, junior physician, and senior physician), and shift types (day, evening, and night).

Having identified key areas of interest and initial findings, we conducted additional semi-structured interviews with eight nurses and two physicians to highlight specific challenges to shift handovers and ongoing assessment of patients. These interviews were recorded, transcribed, and analyzed in Nvivo through a scheme consisting of opencoding and a priori defined codes based on our growing understanding of the context. We used this step as respondent validation and as a further expansion of our data triangulation (Barbour 2001).

In (Singer et al. 2008), a range of qualitative research methods for use in empirical studies is described; these provided guidance in designing a protocol that sought to minimize the risk of influencing the environment with our presence. Thus, we designed a methodology, in which the first author collected information through participant observation as described by Taylor and Bogdan (1984) as "research that involves social interaction between the researcher and informants in the milieu of the latter, during which data are systematically and unobtrusively collected." In our setting, this translated into the main author, who has no clinical expertise, suiting up as a nurse and following several clinicians during their workday. Participatory observation was used as the framework into which we captured information via other instruments such as investigator observations, think aloud observations, and open-ended interview questions. Notes were taken throughout each shift by registering the time, action, and frequency of occurring events.

During the field study observations, we had the opportunity to observe and potentially engage with all communication elements of the work place. After each session, the observation notes were transcribed and the clinician was given the opportunity to make revisions if needed, although this was not the case in any of our sessions. The approved observation notes were analyzed in Excel by the authors following an iterative approach which ultimately identified the units of analysis listed in Table 1. The occurrence of each unit was identified in the notes and summarized.

Our presence at the department gave us access to observe and interact with IT systems and to get copies of the various artifacts in use by the clinicians. Furthermore, the main author participated in staff meetings, triage training, and workshops on patient monitoring. In addition to the field study approach, all heart rate, arterial oxygen saturation, respiration rate, and blood pressure vital signs from the CAP department were also registered in a local research database.

Approval for the study was given by the ED management group, and all involved clinicians consented in our presence. As we had no direct interaction with patients and registered no patient-specific information during the observations, no patient approval was needed. The procedure of our study was presented to the Scientific Ethics Board of southern Denmark, but did not require approval as there were no interventions. The registration and analysis of vital sign values were approved by the Danish Data Protection Agency and conducted in compliance with Danish legislation.

At the time of writing, this database contained vital values from more than 6,700 patients, collected in the period October 2013-December 2014.

\section{Findings}

Table 1 summarizes the field study observations concerning interactions in the JED between clinicians and tools, patients, locations, and colleagues. The numbers in each cell indicate the average number of observations for the given event. Table 1 illustrates how interaction frequency in relation to fellow clinicians, information systems, and patients vary substantially. In the following subsections, we will refer to Table 1 multiple times, and thus defer further explanation for now.

\subsection{Bossen's seven parameters}

\subsubsection{The degree of distribution}

The physical distribution of clinicians heavily impacts how information is created and knowledge is shared. At the ground floor, we find the physician command center, where both senior and junior physicians coordinate and practice much of their work. This resembles a control room where operators keep track of status and flow of a system. Team nurses (the CAP and ETC nurses) are confined to an isolated context, whereas the coordinating nurse spans multiple teams and wards. The degree of distribution of workers in the department is thus heavily related to its infrastructure and the role of each worker.

One of the most noticeable observations from Table 1 is the solitude of the triage nurses. During our observation session, the triage nurse had next to no interaction with clinical colleagues. Given the importance of accurate triage classification, the department seeks to counter this lack of co-present coordination through strict formalization and frequent training to align the classification process. Yet, we still encountered many cases of physicians questioning why a given triage level had been decided upon. This could partly be deduced from the ECP, still the system as a whole lacked a mechanism for expressing this information in relation to the patient-specific boundary objects. 
Table 1 Observed interactions in the JED

\begin{tabular}{|c|c|c|c|c|c|c|c|}
\hline \multirow{2}{*}{$\begin{array}{l}\text { Clinical role and number of } \\
\text { observation sessions }\end{array}$} & \multicolumn{7}{|c|}{ Average times per 8 -h observation session the subject } \\
\hline & $\begin{array}{l}\text { Used } \\
\text { IT }\end{array}$ & $\begin{array}{l}\text { Was present } \\
\text { in office }\end{array}$ & $\begin{array}{l}\text { Was with } \\
\text { patients }\end{array}$ & $\begin{array}{l}\text { Used their } \\
\text { phone }\end{array}$ & $\begin{array}{l}\text { Interacted } \\
\text { with nurses }\end{array}$ & $\begin{array}{l}\text { Interacted with } \\
\text { physicians }\end{array}$ & $\begin{array}{l}\text { Interacted with } \\
\text { assistants }\end{array}$ \\
\hline CAP nurse ( 2 sessions) & 13 & 12 & 31 & 7 & 10 & 7 & 8 \\
\hline CAP assistant ( 1 session $)$ & 8 & 5 & 30 & 8 & 16 & 3 & 3 \\
\hline $\begin{array}{l}\text { CAP-COOR-nurse } \\
\text { (1 session) }\end{array}$ & 19 & 43 & 10 & 66 & 41 & 35 & 5 \\
\hline ETC nurse ( 2 sessions) & 10 & 10 & 22 & 8 & 8 & 9 & 0 \\
\hline Triage nurse (1 session) & 24 & 0 & 24 & 7 & 1 & 1 & 0 \\
\hline $\begin{array}{l}\text { Junior physician } \\
\text { (1 session) }\end{array}$ & 28 & 17 & 9 & 4 & 5 & 19 & 0 \\
\hline $\begin{array}{l}\text { Senior physician } \\
\text { (3 sessions) }\end{array}$ & 31 & 17 & 20 & 6 & 9 & 22 & 0 \\
\hline
\end{tabular}

Physicians generally operate in a more distributed manner, by following an operating configuration that resembles a star-like network, and their patterns resemble 'out and back' activities, as each physician would normally only leave the ETC control room to consult a single patient, then return to the control room, assess their observations, and then initiate a new round of 'out and back.' However, we did on a few occasions see physicians who sought to optimize their workflow by pooling patients together. From Table 1, we see that the number of office stays varies a lot with the clinical profile. In roles where coordination is very important, such as for coordinating nurses and senior physicians, the clinician is seen operating with a higher degree of distribution.

The distribution of clinicians and their SOPs are an important matter in relation to noticing patient deterioration. One CAP nurse noted the following when we discussed the internal transfer of patients:

Often when we receive deteriorated patients here at the CAP, such as patients with atrial fibrillation, they have only been monitored very briefly at the ETC, and have thus not been identified as adverse cases.

Thus, we need to spot this situation on our own.

An equally important degree of distribution concerns the placement of patients. The nurses seek to arrange patients in rooms according to their estimated need for care by assigning more demanding patients closer to the ward offices. This both optimizes their work trajectories and increases the overall level of observation for these patients. Figure 3, which is generated from the collected vital signs grouped by bed, depicts how the intensity of patient monitoring on two CAP wards is related to how far the patients are placed from the nurse offices.

Naturally, the nurses wish to place the most critical patients in the vicinity of the offices of each ward which

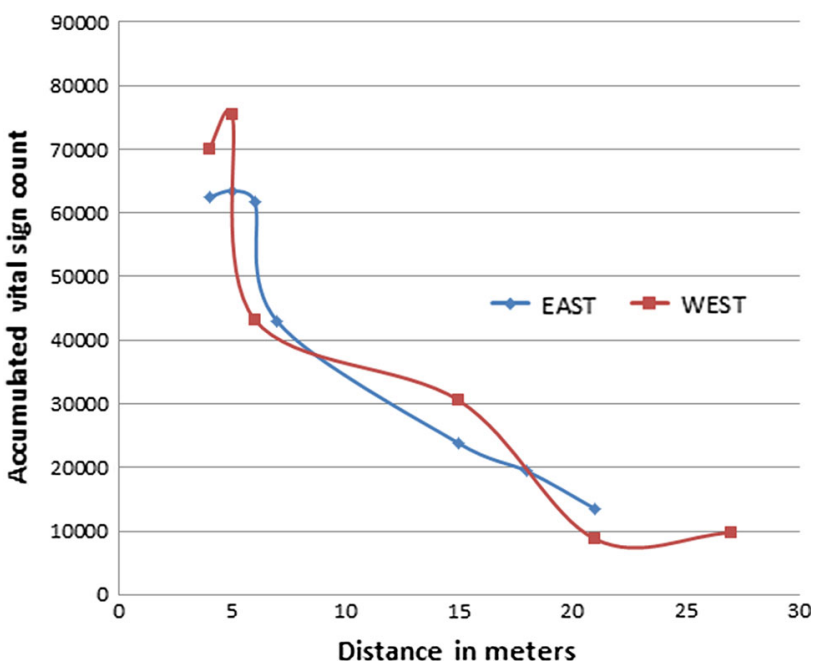

Fig. 3 Relationship between monitoring intensity and distance from nurse offices

acts as communication centers. This caused frequent problems for patients on the four surgical CAP ward beds, which were under the responsibility of the medical wards. Patient observations in these beds were challenged by a lower amount of automated monitoring and longer physical distances. Often these beds were also used for isolation of highly infectious patients. In these cases, nurses had to put on additional safety clothing to prevent spread of infection, and the door to an infected room would always be closed. The danger of this was expressed by a senior physician:

There is nothing more dangerous in the ED than a closed door.

\subsubsection{The multiplicity of webs of significance}

The different backgrounds of assistants, nurses, and physicians make it relevant to examine the multiplicity of 
webs of significance at the department. The time and effort required to enter the professional ranks of these groups scale exponentially, yet all three groups collaborate closely on achieving the same goal, namely the successful treatment of hospitalized patients. This shared mission and the fact that the department was recently established in a manner that required cross-expertise collaboration in new ways, explain why we observed such a high degree of overlap in the webs of significance in terms of willingness to share knowledge and in embracing the knowledge of others into ongoing clinical decision making within the context of each ward.

Besides these formal roles, we also found that the level of individual expertise and competencies impacts the webs of significance, which is in line with Bossen's observations on the necessity of familiarity (Bossen 2002). This is also reflected in Table 1 as each clinical group cooperates most heavily with the same group, showing a need to draw upon the competencies and knowledge present within the same group.

The majority of work place studies of healthcare environments focus mainly on the workers of the organization. Still, the patients are also participants in the CIS and could be linked much closer into the active generation of information as an additional web of significance. Direct patient involvement was, however, outside the scope of our approval, so we deferred any qualitative data collection from patients.

\subsubsection{The level of required articulation work}

The processing of patients in emergency care differs from other departments as patients do not arrive with a diagnosis, but instead with a range of symptoms and a background story (Kovacs and Croskerry 1999). From these pieces, the clinicians attempt to solve the puzzle of determining a probable diagnosis and a suitable treatment within the first $8 \mathrm{~h}$ of presentation, which represents the first phase of a hospital admission. During this phase, the primary articulation work takes place among junior and senior physicians. The ETC nurses spend a lot of their time with patients to inform them of their current status in the diagnosis processing; in this regard, the ECPs greatly relieve them of articulation work and coordination with physicians.

Across all wards at the department, nurses are fundamentally at the front line of observation as they provide both care and administration of medication to patients. From these tasks, and the handling of patient requests, the nurses represent the primary clinical observation channel, whereas physicians are more in contact with patients in points of time through discussions, tests, and observations, which establish the data foundation from which they construct clinical evaluations and diagnosis. From Table 1, we see that caregivers (nurses and assistants) have a much higher bedside presence over number of patients ratio compared to physicians. This was in line with our registration of the pattern that a physician would see a patient and afterwards consult with the attending nurse to consolidate observations and plans.

These parallel but highly cooperative work practices emphasize the high level of required articulation work. Knowing what to do, when to do it, and how to do it, is documented in an elaborate department intranet which contains descriptions of roles, processes, SOPs, and several other artifacts, all for reducing articulation work at the JED. The hospital information systems also reduce the amount of required articulation work, especially in between shifts, where we often observed nurses starting a new shift without any prior knowledge other than that they relied on being available in the EHR. However, as we will discuss shortly, there are downsides to this reliance of articulation work reduction, which can directly impact the identification of deteriorating patients.

Of the utilized vital sign sensors, the blood pressure cuff imposes the most discomfort to the patient, so frequently the caregivers only attach these cuffs when the measurements are taken.

Figure 4 shows the percentage of total blood pressure measurements taken by hour of day. The figure displays measurements for two periods of 79 days each [before (October 1, 2013-December 18, 2013); after (October 1, 2014-December 18, 2014)]. Before and after the department management introduced more rigid measurements schemes. The new schemes required nurses to measure vital signs at specific times throughout the day depending on the triage level of each patient. Prior to the change, measurements during the day shift would be postponed until the end. A pattern is directly comparable with the findings of (Hands et al. 2013). Consequently, there was an increased risk of forgetting to act on newly identified deterioration during the impending handover of shifts between clinicians, and we focus on these challenges in Sect. 6.1.6. The new scheme triggered spikes during the start of each shift, but initial experiences with the more rigid measurement scheme were positive, as stated during one of our interviews:

The new system has greatly improved how good nurses are at remembering to monitor patients according to their triage level, and their ability to document when they decide not to monitor. 
Fig. 4 Blood pressure registrations by hour of day

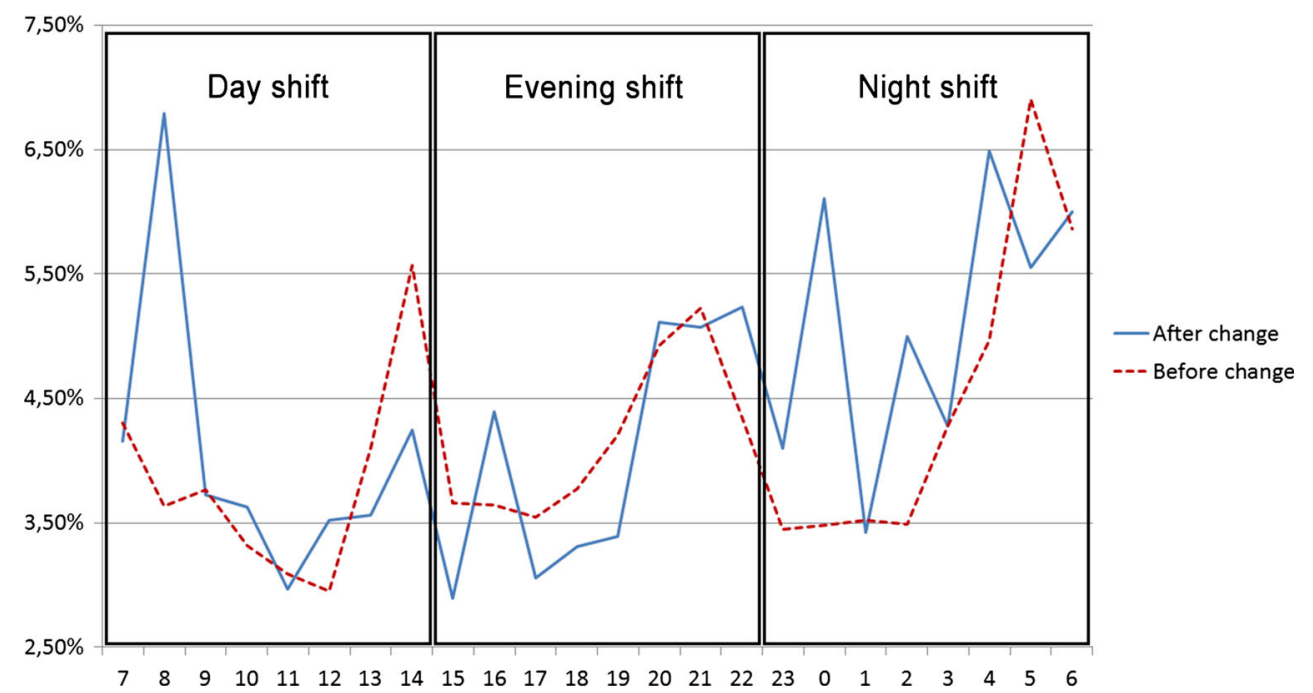

\subsubsection{The multiplicity and intensity of means of communication}

The need for coordinating activities is most present in the two largest groups of employees in the JED: nurses and physicians. Physicians meet twice daily: initially at the morning conference at $8 \mathrm{a} . \mathrm{m}$. in the meeting room facilities at CAP, where all medical patients are reviewed in turn, and the attending physician explains any ongoing treatment, prognosis, and possible discharge/transfer plans. And, again later in the afternoon at 3.30 p.m. when the day shift is relieved, and all patients are reviewed once again, this time in the control room at the ETC. The nurse teams do not meet formally on a daily basis, but rather carry out ad hoc reporting individually when the shift ends, but this seems to vary a lot from nurse to nurse. Handovers are identified as essential events in ensuring a steady continuity of care and patient safety (Randell et al. 2010). Randell et al. also highlight the importance of establishing systems which support collaborative work in terms of knowledge transfers during handovers. Sensible cognitive artifacts, which visualize important information, are proposed as means to reduce the importance of formalized handovers.

The coordinating nurses from each floor meet twice a day with the ETC flow master to review the patients who are scheduled for internal transfer between the ETC and the CAP. Hence, there are several formal structures present for exchange of knowledge, but the most important exchange of information, which is the one that takes place between clinicians and patients, is much harder to formalize. A coordinating nurse on the CAP explained the implications of not being able to observe the patients freely and communicate unhindered with them:

It's often during nighttime we experience that patients deteriorate. It can happen while they sleep, and we do not notice it until it is time for us to do the regular vital sign measurements.

Figure 5 shows how clinicians seek to overcome this challenge by increasing monitoring of patients during the nighttime hours. The majority of nighttime vital signs are $\mathrm{SpO} 2$ and pulse rate measurements as these are the least invasive, but also associated with the highest degree of uncertainty and noise (Elliott and Coventry 2012).

During our second round of interviews, we discussed the option of introducing a more advanced patient feedback system. All clinicians acknowledged that the expressiveness of patients constitute a major aid in ability to assess the state and progression of the patients. However, several clinicians also noted the obvious potential problems with a system, which enable patients to provide direct feedback on their own perceived state:

I'm not sure how valid information from such a system would be because it's highly subjective. I prefer to ask them myself.

Some were more optimistic about the idea:

I think it would be an advantage. Many patients misuse the bell cord (for calling nurses) today, and a more nuanced level of alerting options would help us prioritize our work.

\subsubsection{The web of artifacts}

The notion of a web of artifacts covers both the actual artifacts, also known as the material mechanisms of interaction as described in (Schmidt and Bannon 1992), and how they are utilized through coordination mechanisms, which describe the relationship between protocol of use, and the artifact itself, as proposed by Schmidt and Simone (1996). 


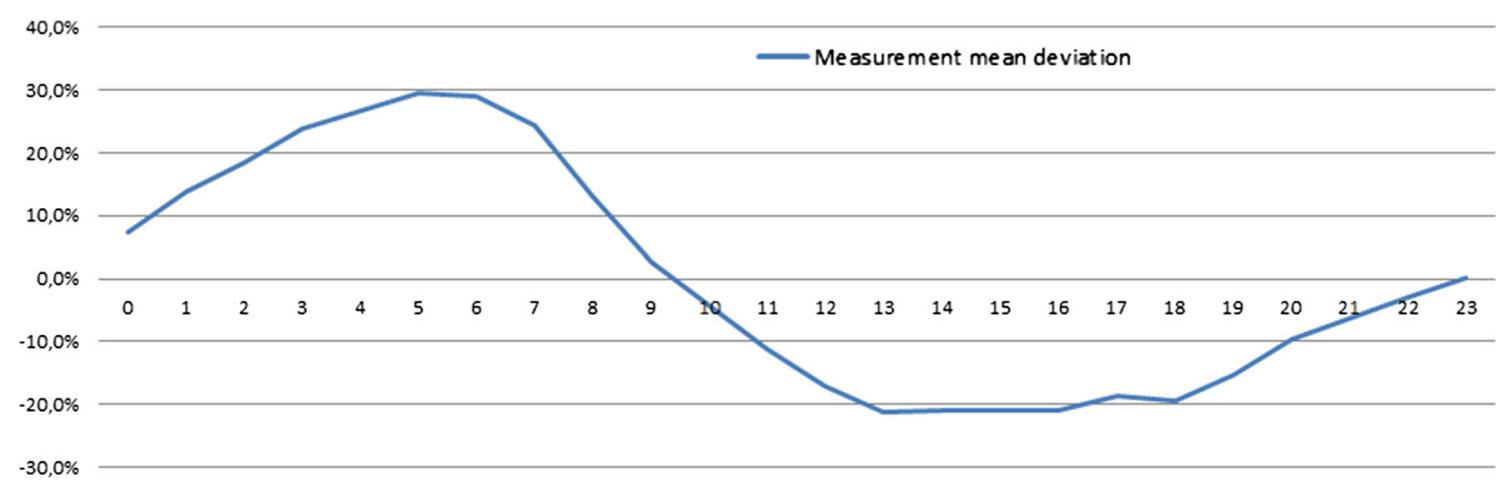

Fig. 5 Monitoring intensity by hourly deviation from mean

Danish hospitals are approaching a paperless system, as records, medication plans, laboratory tests, and images are stored electronically. Even so, a number of paper-based forms are still at play during the admission processing of patients. In our case, two paper-based forms are of interest, as they serve the purpose of recording information about patients until it can be entered into the hospital's information systems. The flow master registers incoming patients on an orange paper form, noting down enough information to uniquely identify the incoming patient, reason for admission, concerns, and needed medical checks. The other paper-based artifact is a green paper form with exactly the same information fields, but instead used by the coordinating nurses to register data about internal patient transfers. Both of these forms are utilized in an immutable way; i.e., no further registration takes place after initial use of the form.

We also observed a continued need to utilize material artifacts for personal coordination, as both physicians and nurses construct individual systems to aid them in keeping track of patient-related information. Nurses would establish their personal artifacts based on predefined information schemas which they then populated in different ways. Physicians relied more on personal notebooks which they structured in individual systems, but often indexed using stickers with patient ID information. These individual systems could be seen as indications of shortcomings for the formalized coordination mechanisms for keeping track of patients, which is in line with the need to modify information interfaces (Xiao et al. 2002).

It also seems that the ability to process and assimilate new information is coupled to the clinician's individual adeptness in autonomously modifying their surroundings and supporting tools to fit their needs through reconfiguration. As an example, we on several occasions observed nurses with previous experience from ICUs modifying and tweaking the patient monitors and their alarm limits, to reduce both the false-positive alarm rate and the number of needed patient-related interventions.

\subsubsection{Immaterial mechanisms of interaction}

This parameter concerns the mechanisms which reduce the need for continuous coordination as the interpretation of a given work setting can be based on minimum units of information. In our setting, we find examples of such mechanisms mainly in the group of CAP nurses as their surroundings are the most stable in the department. This comes to show in the undocumented, but strikingly similar routines of nurses on all three CAP wards as they usually start their shift by familiarizing themselves with patients and then proceed to the administration of medication, and so forth. Albeit this familiarization process is important, it also poses a risk to the patients, as noted by a senior physician:

What frequently happens is that we have an hour, e.g., between 3 p.m. and 4 p.m., where nothing really happens as most clinicians focus on getting up to speed with their patients' history. This yields a period where critically ill patients receive less attention.

Although studies on patient safety emphasizes the importance of handovers (Apker et al. 2007), our second round of interviews found that this practice was highly individual. Basically, the department deals with three fundamental types of handovers: inter-department from the ETC to the CAP, shift handovers, and transfer handovers to other departments. Although handovers to other departments are particular error prone (Frankel et al. 2012), our study focuses on deterioration in the JED. Hence, we only looked at inter-department and shift handovers. In line with the statement in Sect. 6.1.1, a nurse noted that:

When I receive patients from the ETC and start measuring their vital signs, they often tell me that the nurses just did this downstairs. Then, I tell them that they're now in my custody, and because I don't know their state, I need to do it again. 
The format of shift handovers depended a lot on individual clinicians, but also the severity of their patients. Several nurses noted that if they had ill patients they would inform the substituting colleague of their patients to ensure attention during the handover period. Additionally, the structure of handovers depended on the preferences of the participants:

There is no overlap between shifts, but often we either get there a bit earlier, or stay a bit longer to share a few words on the patients.

This handover pattern establishes a joint focus of attention between the outgoing and incoming nurses to reduce the likelihood of overlooking crucial information during the shift transition (Sujan et al. 2015). Still the handover patterns were very dependent on each individual clinician:

We deliver information orally, but some prefer to only get this information, if it is not available in the EHR, otherwise they will rather read up on the patients on their own.

The ETC environment is more chaotic, as admission load can change unpredictably from hour to hour. In this setting, the immaterial mechanisms of interaction are the CIS itself and not the implicitly established routines as is the case in the CAP. Still, a priori knowledge of standardized care packages and the overall structure of the patient admission plan help the clinicians reduce the need for continuous coordination here as well. If coordination is needed, it is often mediated by the flow master or the coordinating nurse, or by the use of the internal phone system which, as given in Table 1, was used more heavily by some groups than others.

\subsubsection{The need for precision and promptness of interpretation}

Although Bossen groups the notion of precision and promptness into the same parameter, they embody distinct parts of clinical practices. In the JED, preciseness depends on both how well clinicians are able to communicate knowledge about patients to fellow clinicians and also how skilled they are in interpreting the flows of data from patients and their monitoring systems. On multiple occasions, we observed nurses muting patient monitoring alarms, based on either assumptions about the patient, or the equipment. This is in line with the possible causes for alert fatigue as summarized in the literature review by Sijs et al. (2006), who lists causal factors such as a high number of false positives, usability issues, and faith in own knowledge. The monitoring systems issue alarms in a range of levels, and although the most severe alarms have a higher observation rate, inexperienced nurses may be affected by the overall tendency to dismiss non-critical alarms, and thus miss true adverse events on the long term.

The cognitive challenge that occurs when clinicians are faced with situations that conflict with their initial assumptions can also lead to situations of alarm fatigue. We saw an example of this in a situation with a patient whose blood pressure was notably above the accepted threshold, initially the attending nurse tried to solve the problem by changing equipment, but after repeated alarming measurements, and attempts to improve precision of measurement, discussions with a colleague clarified that high blood pressure was normal for the patient. This incident illustrates both the poor specificity of alarms generated from population-based thresholds and the need for a patient monitoring system that adapts to more specific traits. These traits can be both group-specific traits and characteristics of the individual patient.

Promptness is the ability to respond in an appropriate manner to a given situation. If the patient suddenly deteriorates severely, and the deterioration is detected, a medical emergency procedure is initiated; this involves moving the patient to a special trauma room, which is then manned by physicians' and nurses' on-call for such events. These teams are all trained in simulated scenarios and have well-established SOCs and SOPs in place. In one of our observation sessions, we observed how an elderly female patient arrived in an already severe state, triage level orange (triage 2 in Fig. 2), and was promptly attached to a monitor as prescribed by procedure. Within minutes of arrival to a normal observation room, blood pressure and heart rate dropped significantly while the attending nurse was present. The coordinating nurse arrived within minutes of the first heart rate alarm and swiftly contacted a senior physician who immediately issued a medical emergency call. The patient was transferred to a trauma room and fortunately stabilized. This incident had a positive outcome because the precision of the initial triage was correct and because all attending clinicians responded promptly. Even so, it shows the crucial importance of accurate representation of patients and continuous monitoring.

\subsection{Review of existing patient monitoring platforms}

To complement the findings from our field study, we have conducted a non-formal review of current patient monitoring platforms from 15 different manufacturers. The intent was to build an aggregated feature list, which we used for comparison with our field study findings. The list is presented as a taxonomy for patient monitoring solutions.

We restricted our review to only include multivariate monitoring platforms, and thus excluded devices 
Table 2 Patient monitoring taxonomy

\begin{tabular}{|c|c|}
\hline \multicolumn{2}{|c|}{ Patient monitoring taxonomy } \\
\hline Continuum of care & Concerns the transfer and handover of patients across beds and departments \\
\hline Historical data access & Does the system support storage and retrieval of historical data? \\
\hline Mobile monitoring & To what extent does the system support continuous monitoring during transfers and handovers? \\
\hline $\begin{array}{l}\text { Mobile unit } \\
\text { notification }\end{array}$ & Does the system support delivery of notifications and events on mobile units? \\
\hline Collaboration & Concerns support for knowledge sharing and collaboration between clinicians. \\
\hline Inter-bed inspection & Is support provided for retrieving patient information and alarms on other bedside monitoring units? \\
\hline $\begin{array}{l}\text { Knowledge } \\
\text { facilitation }\end{array}$ & Does the platform support registering and mediating knowledge between clinicians? \\
\hline Central monitoring & To what extent does the platform support remote monitoring on central monitoring stations? \\
\hline SOP support & Does the platform provide a framework for codifying procedures or clinical rules directly into the unit or platform? \\
\hline Integration & $\begin{array}{l}\text { Concerns the support for integration with third-party systems and the ability to share and retrieve information across } \\
\text { units }\end{array}$ \\
\hline HIS integration & Does the system provide support for exporting and retrieving information from other HIS setups? \\
\hline Networked & Does the system support a networked architecture? \\
\hline Other bedside devices & Can the system include data from other bedside devices directly? \\
\hline Mounting & Concerns how the patient monitoring unit is mounted by the bed and how it communicates with other devices \\
\hline Placement & How is the device mounted? Typically portable on bed or stationary on a stand \\
\hline Connections & How does the system communicate with sensors and other devices? Typically cabled or wireless \\
\hline Alarms & Concerns the patient monitoring platform's ability to convey information \\
\hline $\begin{array}{l}\text { Configurable } \\
\text { thresholds }\end{array}$ & To what extent does the system support configurable thresholds for vital sign alarms? \\
\hline Alarm types & What types of alarms are supported by the device? Typically sensory, visible, and audible alarms \\
\hline EWS & Does the device include public or proprietary EWS systems or other aggregated scoring? \\
\hline Sensors & Concerns the range of sensors supported by the patient monitoring platform \\
\hline Types & What range of sensor types are supported by the patient monitoring platform? \\
\hline User interfaces & Concerns how the unit conveys information about patient vitals \\
\hline Presentation mode & To what extent does the system support multiple screens? \\
\hline Interaction & What types of interaction are supported? Typically touch screens, buttons, and dials \\
\hline Customization & How flexible is the system in its ability to rearrange and customize displayed content? \\
\hline Trends & Does the system support displaying vital signs as trend lines? \\
\hline Figures & How does the system support the ability to display vital signs as figures? \\
\hline
\end{tabular}

specialized for specific physiological parameters. We collected data on existing devices by inspecting the available product pages and datasheets from each company's product portfolio and register all listed features. Several manufacturers offered multiple solutions, but as we were more interested in capabilities than specific products, we only registered features on a manufacturer level. The complete list of features was then reviewed, and common themes were extracted as categories.

After identifying common themes, we returned to the complete list of features and established a set of parameters which covers features associated with each theme. The themes and associated parameters are presented in Table 2. This taxonomy will help us to assess the novelty of our field study findings in the later Discussion.
Few of the major manufacturers offer built-in support for automated EWS tracking. The latest Philips IntelliVue monitors provide the Guardian Early Warning Score, which supports both Single-Parameter Scoring (Smith et al. 2008) and MEWS. The OBS Medical Visensia platform, which is based on BioSign, offers the most advanced means for automatically calculating a severity score (Orphanidou et al. 2009).

A systematic review of patient monitor displays reported a positive effect of integrated graphical displays (Görges and Staggers 2008). The same review also found that most commercial systems inadequately include the workflow and needs of nurses into their design. In general, the platforms offer varying degrees of support for collaborative work and situation awareness. Some of these aspects alleviate issues such as knowledge facilitation and better HIS 
integration with patient deterioration detection at the JED of this study. Other identified issues appear unsupported across all platforms, as we will review in the 'Discussion' section.

\section{Discussion}

It is evident that the CIS of the JED in our field study is a complex interplay of people, places, and artifacts. In this distributed environment, knowledge of each patient's state is essential, yet the system only works if this knowledge is distributed in accordance with the fact that individual clinicians strive to focus only on subsets of the accumulated shared knowledge, while seeking to streamline their own work flows to remove as many cognitive friction points as possible. Other friction points are cases of knowledge being stuck, such as having to wait for the transcription of physician observations to the EHR, which can severely hinder the processing of patients. This obstacle can be abstracted to other levels, as well as to situations where past actions are not articulated in the common space. In relation to detecting patient deterioration, this is most evident in the present information system's inability to convey the alarm history and progress to clinicians, which is amplified in effect when clinicians forget to transfer knowledge between shifts.

As patients of all triage levels are at risk of deterioration, the less critical patients, who are often both placed further away from the ward nurse offices and monitored considerably less, would seem to be at higher risk of un-noticed deterioration. Likewise, patients who deteriorate during night shifts are generally more likely to remain undetected despite the overall increased level of monitoring. Correspondingly, systemic improvements to patient deterioration detection should encompass both spatial and temporal aspects. Patient observation and monitoring are challenged differently depending on the time of day, including the patients more directly into the CIS could mitigate the risk of clinicians not being at the right place at the right time, which in our case is prior to the time of deterioration onset. As reviewed earlier, this inclusion is not trivial and perhaps the solution would be to incorporate additional information captured automatically from patients.

From a systemic level, one needs to acknowledge the fact that the CISs of today's hospital departments are built on a partnership between human and technology driven subsystems, and for the whole to operate optimally, we need to consider each part individually and together. With regard to detecting adverse events, precision in classification stands out as a key factor in establishing a baseline from which deviation is to be determined. Instead of basing the deviation detection on population-based models, it may be feasible to construct models of normality in a real-time perspective (Zhang et al. 2007). Additionally, to embed the notion of time is rarely included into current clinical models (Kennedy and Turley 2011). Although changes and not only absolute values in physiological parameters have been identified as important (Kellett et al. 2013), it is surprising that no EWS system seems to include any deviance from previously recorded values in their aggregated scoring models as both time and direction of trajectory are of importance.

The use of artifacts and systems in handovers vary a lot between clinical groups. While the process is standardized for physicians and coordinating nurses (Wears et al. 2006), it is less structured for department ward nurses. But imposing a more rigorous handover process may simply add more cognitive load upon the nurses. Instead, it may be favorable to emphasize patient-centric artifacts which can transcend the moment of handover.

Also, expert system capable of replacing the clinical sixth sense is still a far-fetched futuristic scenario and probably not a very desirable one either. Thus, in line with the work of (Kane and Luz 2009), we find that one must consider the impact a given technology has on the process of identifying patients at risk of deterioration. It would seem that the current system in use at the JED does not fully provide the necessary range of support for the spectrum of clinical competencies which Kane and Luz concluded was important to maximize the gain of having multiple specialties at hand.

Such a collaborative deterioration platform should also seek to embed support of 'professional vision' as defined by (Goodwin 1994) as the representation of (1) coding of phenomena and their transformation into objects of knowledge, (2) emphasis on specific progression phenomena to draw them out in their complex surrounding field, and (3) how material objects are produced and articulated. The artifact centeredness on patients is congruent with the clinical mind-set. But, the representation of a patient's trajectory and state is lacking.

In summary, the observations offer guidance in designing an automated patient deterioration warning system, which (1) builds upon patient-specific models of normality, (2) provides support for integrating patient provided input, (3) presents the state of patients ubiquitously across time and space, and (4) supports artifactual multiplicity of patient representation to enable knowledge transfer between clinicians.

Comparing these proposals with the taxonomy proposed in Table 2, we find that:

1. Patient-specific models of normality: Most existing monitors support a wide range of customization options and structures of patient profiles. However, none of these automatically adapt to the specific patients, but 
instead rely on population-based assumptions. An often raised critique of EWS systems is their lack of proper clinical validation (Cuthbertson and Smith 2007). To deal with this issue, machine learning research is gradually making its way into patient monitoring and adverse event detection (Escobar et al. 2012; Hackmann et al. 2011). However, we have not found any systematic reviews on the use of machine learning in patient monitoring. The majority of attempts are still at a very early stage, although some large-scale validation studies have been performed (Clifton et al. 2013). Based on our field study findings, and the exploration of published research, we argue that there is a need to investigate the potential for building a system, which supports patient-specific models of normality, and to systematically review the application of machine learning to identification of patient deterioration. We propose a three-staged approach which gradually evaluates patient deterioration given available information (Schmidt and Wiil 2015).

2. Support for patient provided input: Although vital signs are considered as patient-related input, we find that no patient monitoring platforms provide support for subjective patient input. Several of the scoring systems include metrics based on either clinical observations or patient input, such the AVPU metric in ViEWS (Prytherch et al. 2010) or pain scales. Although the subjectivity of these parameters present both communication and interpretation challenge, the right tools may increase the likelihood of positive clinical utility (Williamson and Hoggart 2005). We propose that identifying ways of embedding pain, or other discomfort features into patient monitoring, would help identifying patients at risk of deterioration, with the consideration that such a feature should not be thought of as an alternative to traditional nurse calls.

3. Ubiquitous representation of patient state across time and space: Sensible cognitive artifacts can aid in a better awareness and thus a deeper understanding and immersion in the CIS (Randell et al. 2010). We find several solutions in existing monitors for ensuring the availability of patient information when and where it is needed through inter-bed inspection, notifications, and central monitoring stations. Yet, few of them have built-in adaptation to temporal challenges; e.g., it may be preferable to automatically simplify the displays during handovers. Additionally, we have still to find a patient monitoring platform which is capable of communicating information about patients to clinicians regardless of location. One way to deal with this challenge is using either augmented reality or other pervasive technologies (Sanderson et al. 2005). Most current research on the use of such technologies focuses on the application within closely situated contexts such as operating rooms. Thus, we find that there is a need to investigate the usefulness of such tools in a more distributed and diverse clinical setting such as an ED.

4. Artifactual multiplicity of patient representation: The need for personal cognitive artefacts representing patients was observed across all clinical professions. However, the clinical utility of such artifacts is closely related to malleability, ownership, and accessibility (Wears et al. 2006) and (Xiao et al. 2002). Supporting artifactual multiplicity as defined by (Bjørn and Hertzum 2010) must address the heterogeneity of clinical practices, while still accommodating the different clinical realities and circumstances present in each clinical profession. Patient monitoring is an important tool for sharing situation awareness between closely located clinicians (Schulz et al. 2013), but most current patient monitoring technology mainly represents patients through visualization of numbers or trends at the bedside or via central monitoring stations. We argue that this approach in no way supports the collaborative needs of a clinical CIS.

Elaborating upon each of these four areas will help us gain a better understanding of areas of patient monitoring which are currently largely uncharted.

\section{Limitations}

External validity in this study has mainly relied on generalizability through transferability of our findings. As a mean to achieve this, we have provided a thick description of the settings (Polit and Beck 2010). Alternatively, additional or other layers of validity could have been applied to this study by seeking other means of generalization such as a more statistical approach. However, this would have required us to limit the broadness of our study scope, and we would not have been able to cover the variety of data sources that we felt was needed to convey the richness of the research question.

\section{Conclusion}

The main contribution of this paper is our identification of possible areas of improvement for future deterioration detection systems. Our field study has exposed the complexity of the CIS in a particular JED, and based on the findings and discussion in this paper, we see multiple paths opening up for future work. Returning attention to our research question and how CIS have aided us in finding 
answers, we find that the need for articulation work is related to the clinical risk assessment of each patient. Patients at risk incite the clinicians to take extra measures in ensuring that information is delivered immediately. Organizational aspects such as handover routines, department infrastructure, and division of work emerged during our study. We have affirmed the importance of enabling the clinicians to customize their working environment, especially their IT tools.

Our review of technical aspects hindering identification of patients at risk of deterioration mainly focused on issues directly related to patient monitoring. The present systems have specific shortcomings such as no representation of patients outside the offices, no differentiation in representation of state to different clinical groups, and a lack of patient trajectory visualization. These shortcomings vary in both temporal and spatial manner. The findings are important to our future work with regard to designing patient deterioration detection prototypes which are viable not only on a clinical level, but also with regard to frictionless embeddedness into everyday work practices.

We also proposed a taxonomy for the evaluation of patient monitoring systems to support our findings. More systematic future work is needed to evaluate and validate this taxonomy.

Acknowledgments We would like to thank the staff members and researchers from Fælles Akut Modtagelsen at Odense University Hospital for their collaboration and enthusiasm in participation, especially Annmarie Touborg Lassen, MD, Professor, Ph.D. DMSci, Michael Hansen-Nord, Chief Consultant, Charlotte Mose Hansen, Head nurse, Marianne Glud and Susanne Ravn, Nursing head of units. This project is a part of Patient@home (www.patientathome.dk).

Open Access This article is distributed under the terms of the Creative Commons Attribution License which permits any use, distribution, and reproduction in any medium, provided the original author(s) and the source are credited.

\section{References}

Apker J, Mallak LA, Gibson SC (2007) Communicating in the "gray zone": perceptions about emergency physician hospitalist handoffs and patient safety. Acad Emerg Med 14:884-894

Bannon L, Bødker S (1997) Constructing common information spaces. In: Proceedings of the fifth european conference on computer supported cooperative work. Springer, Netherlands, pp 81-96

Barbour R (2001) Checklists for improving rigour in qualitative research: a case of the tail wagging the dog? BMJ 322:1115-1117

Bardram JE, Bossen C (2005a) Mobility work: the spatial dimension of collaboration at a hospital. Comput Support Coop Work 14:131-160

Bardram JE Bossen C (2005b) A web of coordinative artifacts: collaborative work at a hospital ward. In: Proceedings of the 2005 international ACM SIGGROUP conference on Supporting group work. ACM, pp 168-176
Bjørn P, Hertzum M (2010) Artefactual multiplicity: a study of emergency-department whiteboards. Comput Support Coop Work 20:93-121

Bjørn P, Rødje K (2008) Triage drift: a workplace study in a pediatric emergency department. Comput Support Coop Work 17:395-419

Bossen C (2002) The parameters of common information spaces: the heterogeneity of cooperative work at a hospital ward. In: Proceedings of the 2002 ACM conference on computer supported cooperative work. ACM, pp 176-185

Brabrand M, Folkestad L, Clausen NG, Knudsen T, Hallas J (2010) Risk scoring systems for adults admitted to the emergency department: a systematic review. Scand J Trauma Resusc Emerg Med 18:8

Brabrand M, Hallas J, Knudsen T (2014) Nurses and physicians in a medical admission unit can accurately predict mortality of acutely admitted patients: a prospective cohort study. PLoS ONE 9:e101739

Brady PW, Muething S, Kotagal U, Ashby M, Gallagher R, Hall D, Goodfriend M, White C, Bracke TM, DeCastro V, Geiser M, Simon J, Tucker KM, Olivea J, Conway PH, Wheeler DS (2013) Improving situation awareness to reduce unrecognized clinical deterioration and serious safety events. Pediatrics 131:e298e308

Clifton DA, Wong D, Clifton L, Wilson S, Way R, Pullinger R, Tarassenko L (2013) A large-scale clinical validation of an integrated monitoring system in the emergency department. IEEE J Biomed Health Inform 17:835-842

Cuthbertson BH, Smith GB (2007) A warning on early-warning scores! Br J Anaesth 98:704-706

Dawes TR, Cheek E, Bewick V, Dennis M, Duckitt RW, Walker J, Forni LG, Venn R (2014) Introduction of an electronic physiological early warning system : effects on mortality and length of stay. Br J Anaesth 113:1-7

Elliott M, Coventry A (2012) Critical care: the eight vital signs of patient monitoring. Br J Nurs 21:621-625

Escobar GJ, LaGuardia JC, Turk BJ, Ragins A, Kipnis P, Draper D (2012) Early detection of impending physiologic deterioration among patients who are not in intensive care: development of predictive models using data from an automated electronic medical record. J Hosp Med 7:388-395

Firestone WA (1993) Alternative arguments for generalizing from data as applied to qualitative research. Educ Res 22:16-23

Fitzpatrick G, Ellingsen G (2013) A review of 25 years of CSCW research in healthcare: contributions, challenges and future agendas. Comput Support Coop Work 22:609-665

Frankel RM, Flanagan M, Ebright P, Bergman A, O'Brien CM, Franks Z, Allen A, Harris A, Saleem JJ (2012) Context, culture and (non-verbal) communication affect handover quality. BMJ Qual Saf 21(Suppl 1):i121-i128

Gao H, McDonnell A, Harrison DA, Moore T, Adam S, Daly K, Esmonde L, Goldhill DR, Parry GJ, Rashidian A, Subbe CP, Harvey S (2007) Systematic review and evaluation of physiological track and trigger warning systems for identifying atrisk patients on the ward. Intensive Care Med 33:667-679

Goodwin C (1994) Professional vision. Am Anthropol 96:606-633

Görges M, Staggers N (2008) Evaluations of physiological monitoring displays: a systematic review. J Clin Monit Comput 22:45-66

Hackmann G, Chen M, Chipara O, Lu C, Chen Y, Kollef M, Bailey TC (2011) Toward a two-tier clinical warning system for hospitalized patients. AMIA Annu Symp Proc 2011:511-519

Hands C, Reid E, Meredith P, Smith GB, Prytherch DR, Schmidt PE, Featherstone PI (2013) Patterns in the recording of vital signs and early warning scores : compliance with a clinical escalation protocol. BMJ Qual Saf 22:1-8 
Henriksen DP, Brabrand M, Lassen AT (2014) Prognosis and risk factors for deterioration in patients admitted to a medical emergency department. PLoS ONE 9:e94649

Johansson B, Hollnagel E (2006) Pre-requisites for large scale coordination. Cogn Technol Work 9:5-13

Kane B, Luz S (2009) Achieving diagnosis by consensus. Comput Support Coop Work 18:357-392

Kellett J, Emmanuel A, Deane B (2011) Who will be sicker in the morning? Changes in the simple clinical score the day after admission and the subsequent outcomes of acutely ill unselected medical patients. Eur J Intern Med 22:375-381

Kellett J, Wang F, Woodworth S, Huang W (2013) Changes and their prognostic implications in the abbreviated VitalPAC ${ }^{\mathrm{TM}}$ Early Warning Score (ViEWS) after admission to hospital of 18,827 surgical patients. Resuscitation 84:471-476

Kennedy CE, Turley JP (2011) Time series analysis as input for clinical predictive modeling: modeling cardiac arrest in a pediatric ICU. Theor Biol Med Model 8:40

Kovacs G, Croskerry P (1999) Clinical decision making: an emergency medicine perspective. Acad Emerg Med 6:947-952

Lauritzen M, Skriver C, Dahlin J (2009) Triage-manual. http://www. hillerodhospital.dk/NR/rdonlyres/D20F6C68-ABB6-402D-B463C7293185C372/0/Triagemaster.pdf

Moon A, Cosgrove JF, Lea D, Fairs A, Cressey DM (2011) An eight year audit before and after the introduction of modified early warning score (MEWS) charts, of patients admitted to a tertiary referral intensive care unit after CPR. Resuscitation 82:150-154

Odell M, Victor C, Oliver D (2009) Nurses' role in detecting deterioration in ward patients: systematic literature review. J Adv Nurs 65:1992-2006

Orphanidou C, Clifton D, Khan S, Smith M, Feldmar J, Tarassenko L (2009) Telemetry-based vital sign monitoring for ambulatory hospital patients. Engineering in medicine and biology society, 2009. EMBC 2009. Annual international conference of the IEEE. pp 4650-4653

Polit DF, Beck CT (2010) Generalization in quantitative and qualitative research: myths and strategies. Int $\mathrm{J}$ Nurs Stud 47:1451-1458

Prytherch DR, Smith GB, Schmidt PE, Featherstone PI (2010) ViEWS-Towards a national early warning score for detecting adult inpatient deterioration. Resuscitation 81:932-937

Randell R, Wilson S, Woodward P (2010) Galliers J (2010) Beyond handover : supporting awareness for continuous coverage. Cognit Technol Work 12:271-283

Royal College of Physicians (2012) National Early Warning Score (NEWS). Royal College of Physicians, London

Sanderson PM, Watson MO, Russell WJ (2005) Advanced patient monitoring displays: tools for continuous informing. Anesth Analg 101:161-168

Schmidt K, Bannon L (1992) Taking CSCW seriously : supporting articulation work. Comput Support Coop Work (CSCW) 1:7-40
Schmidt K, Simone C (1996) Coordination mechanisms: towards a conceptual foundation of CSCW systems design. Comput Support Coop Work 5:155-200

Schmidt T, Wiil UK (2015) A 3-staged approach to identifying patients at risk of deterioration in emergency departments. International conference on health informatics. HealthInf2015

Schulz CM, Endsley MR, Kochs EF, Gelb AW, Wagner KJ (2013) Situation awareness in anesthesia: concept and research. Anesthesiology 118:729-742

Sijs H, Van Der Aarts J, Vulto A, Berg M (2006) Overriding of drug safety alerts in computerized physician order entry. J Am Med Info Assoc 13:138-147

Singer J, Sim S, Lethbridge T (2008) Software engineering data collection for field studies. In: Shull F, Singer J, Sjøberg DIK (eds) Guide to advanced empirical software engineering. Springer, Berlin, pp 9-34

Smith GB, Prytherch DR, Schmidt PE, Featherstone PI, Higgins B (2008) A review, and performance evaluation, of singleparameter "track and trigger" systems. Resuscitation 79:11-21

Strauss A (1985) Social organization of medical work. The University of Chicago Press, Chicago, London

Subbe C, Kruger M, Rutherford P, Gemmel L (2001) Validation of a modified Early Warning Score in medical admissions. QJM 94:521-526

Sujan MA, Chessum P, Rudd M, Fitton L, Inada-Kim M, Spurgeon P, Cooke MW (2015) Emergency care handover (ECHO study) across care boundaries: the need for joint decision making and consideration of psychosocial history. Emerg Med J 32:112-118

Tarassenko L, Hann A, Young D (2006) Integrated monitoring and analysis for early warning of patient deterioration. Br J Anaesth 97:64-68

Taylor SJ, Bogdan R (1984) Introduction to qualitative research methods: the search for meanings. Wiley, New York

Wears RL, Perry SJ, Wilson S, Galliers J, Fone J (2006) Emergency department status boards: user-evolved artefacts for inter- and intra-group coordination. Cogn Technol Work 9:163-170

Weingart SD, Wyer PC (2006) Emergency medicine decision making: critical choices in chaotic environments. McGraw-Hill, New York

Williamson A, Hoggart B (2005) Pain: a review of three commonly used pain rating scales. J Clin Nurs 14:798-804

Xiao Y, Lasome C, Moss J, Mackenzie CF, Faraj S (2002) Cognitive properties of a whiteboard: a case study in a Trauma centre. ECSCW 2001. Springer Netherlands, pp 259-278

Zhang Y, Silvers CT, Randolph AG (2007) Real-time evaluation of patient monitoring algorithms for critical care at the bedside. Engineering in medicine and biology society, 2007. EMBS 2007. 29th annual international conference of the IEEE. pp 2783-2786 